\title{
Proximités : retour sur 25 années d'analyse
}

\section{Proximities: return over 25 years of analysis}

\author{
André TORRE \\ UMR SAD-APT, INRA, Agroparistech, Université Paris-Saclay \\ torre@agroparistech.fr \\ Damien TALbot \\ CRCGM, IAE, Université Clermont Auvergne \\ damien.talbot@uca.fr
}

dans Revue d'Economie Régionale et Urbaine, 2018, n5/6, pp. 917-936

\begin{abstract}
Résumé : Après 25 années de recherche, la question se pose de la pérennité de l'approche de la proximité, mais aussi de sa banalisation et de sa capacité à se renouveler dans le monde des sciences régionales, et compte tenu de son impact dans différentes disciplines des sciences sociales. Cet article a pour objet de faire un retour sur ces 25 années d'analyses, afin d'éclairer les raisons de la réussite de ce programme de recherche, de faire le bilan de ses acquis mais aussi de ses échecs et de revenir sur le chemin parcouru, ses avenues et ses bifurcations. Nous commençons par expliciter les enjeux théoriques, sociétaux et académiques à la base de la naissance de l'Ecole de la Proximité (I), avant de présenter les principaux résultats d'ordre analytique, avec leurs limites et leurs surprises (II), puis les acquis en termes académiques et sociétaux (III), pour aborder enfin les angles morts du programme de recherche et le travail restant encore à réaliser (IV). Nous concluons par quelques interrogations sur l'avenir de ce courant de pensée et sa capacité à se renouveler dans un environnement soumis à de profondes mutations dans le monde réel comme dans le monde des idées.
\end{abstract}

\begin{abstract}
After 25 years of research, we ask the question of the sustainability of the proximity approach, and of its recognition and its renewal capacity in the world of regional sciences, considering its impact on various disciplines of social sciences. This article aims to make an overview over 25 years of analyses, to shed light on the reasons of the success of the research program, and to make an assessment of its outputs but also its failures. We come back to the scientific pathway, its avenues and main bifurcations. We start with the main theoretical, societal and academic stakes at the birth of the Proximity School (I), before presenting the main analytical results, with their limits and their surprises (II). Then we assess about the final gains in academic and societal terms (III), to approach finally the dead angles of the research program and the work still remaining (IV). We conclude by some questioning on the future of the proximity analysis and its capacity to be renewed in an environment subjected to profound transformation, be there in the real world or in the world of ideas.
\end{abstract}

Mots clés : proximité, sciences régionales

Key words: proximity, regional science 


\section{Introduction $^{1}$}

Débouchez les bouteilles et envoyez les cotillons, la proximité a 25 ans! L'entreprise confidentielle du début, lancée par six économistes en mal d'ouverture et de reconnaissance, s'est transformée en aventure collective intense, puis en un champ de recherche aujourd'hui internationalement reconnu, avec ses principes analytiques, son itinéraire méthodologique, ses champs d'application privilégiés et ses auteurs phares. 25 ans plus tard, après les poussées de croissance et la crise d'adolescence, la question se pose de la pérennité de cette approche, mais aussi de sa banalisation et de sa capacité à se renouveler dans le monde des sciences régionales.

De la première réunion dans une petite salle de l'Université de Dauphine à la reconnaissance internationale, il est long le chemin. Difficile de prédire le succès dès cette époque, mais l'aventure humaine émergeait déjà : la convivialité, le plaisir de se retrouver plusieurs fois par an dans différents lieux, ont fondé le fonctionnement du groupe Dynamiques de Proximité, permettant à chacun d'investir pleinement le collectif en toute confiance. Car c'est bien un groupe informel de chercheurs amis qui se constitue et se lance rapidement dans la publication de nombreux ouvrages collectifs (RALLET et TORRE, 1995 ; BELLET et al., 1998 ; GILLY et TORRe, 2000 ; DuPUY et Burmeister, 2003 ; PECQueur et ZimMERMANn, 2004, etc.), et qui très tôt s'ouvre à de jeunes doctorantes et doctorants, dont beaucoup aujourd'hui sont eux aussi des universitaires.

La succession de trois numéro spéciaux de la RERU (BELLET et al., 1993 ; BouBA-OLGA et al., 2008 et celui-ci) consacrés aux développements du programme de recherche démontre cet attachement à l'idée de groupe constitué autour de deux ou trois générations de chercheurs. Cette bienveillance a facilité la production de nombreuses avancées scientifiques, car la prise de risque y était possible, voire souhaitée. Et les débats intellectuels qui en découlaient vifs et profonds. Aujourd'hui, les relations sont probablement plus interindividuelles, au gré des publications communes. Surtout, ce que l'on a rapidement qualifié d'Ecole de la Proximité échappe largement à ses fondateurs, ce qui signe son profond succès. Les publications de ses membres sont maintenant minoritaires comparées à celles émergeant de différentes disciplines ou champs d'investigation, souvent distincts de ceux visés au départ, et majoritairement hors de France.

Cet article a pour objet de faire un retour sur 25 années d'analyses de la proximité, afin d'éclairer les raisons de la réussite de ce programme de recherche, de faire le bilan de ses acquis mais aussi de ses échecs et de revenir sur le chemin parcouru, ses avenues et ses bifurcations. Nous commençons par expliciter les enjeux théoriques, sociétaux et académiques à la base de la naissance de l'Ecole de la Proximité (I), avant de présenter les principaux résultats d'ordre analytique, avec leurs limites et leurs surprises (II), puis les acquis en termes académiques et sociétaux (III), pour aborder enfin les angles morts du programme de recherche et le travail restant encore à réaliser (IV). Nous concluons par quelques interrogations sur l'avenir de ce courant de pensée et sa capacité à se renouveler dans un environnement soumis à de profondes mutations dans le monde réel comme dans celui des idées. 


\section{Pourquoi l'Ecole de la Proximité est-elle née ?}

Etablir un bilan appelle un préalable essentiel : rappeler quelle « feuille de route » se sont tracés les chercheurs du groupe Dynamiques de proximité, qui ont pris l'initiative de créer cette Ecole dans les années 90 . Dès le début, en effet, cette dernière a cherché à se positionner en répondant à de grands types d'enjeux. On peut les diviser suivant trois principales catégories de questionnements, de natures théoriques, empiriques et sociétales et, enfin, académiques.

\subsection{Des enjeux théoriques et analytiques}

Sur le plan théorique, le premier enjeu a consisté à établir des ponts solides entre l'économie industrielle et l'économie spatiale/régionale, en considérant à juste titre qu'un croisement d'approches qui s'ignoraient encore serait fertile (RALLET et TORRE, 1995 ; KRUGMAN, 1995). C'est d'ailleurs le principe même de constitution du groupe de départ: trois économistes régionaux et trois économistes industriels. Du côté industriel, l'objectif est d'intégrer le rôle de l'espace très tôt dans l'analyse, et non a posteriori comme un simple facteur explicatif résiduel des modalités par exemple de l'innovation, de l'organisation de la production et des configurations industrielles observables (DUPUY et GILLY, 1995). Il s'agit d'affirmer l'intuition première : au même titre par exemple que le temps, l'espace compte. Du côté régional ou spatial, l'objectif est de prendre en compte de manière explicite les dimensions d'innovation et les changements productifs importants en cours dans les territoires (KIRAT et LUNG, 1999 ; ZIMMERMANN, 1998).

Dès lors, les travaux initiaux indiquent vouloir s'attacher à l'examen des processus de création de ressources pour dépasser l'approche statique de dotations des facteurs (BELLET et KIRAT, 1998). La compréhension de tels processus suppose de faire appel à divers concepts issus des sciences sociales : le pouvoir, la confiance (DUPUY et TORRE, 2004) ou encore les institutions en sont des exemples paradigmatiques. L'établissement de tels liens théoriques suppose d'introduire des approches hétérodoxes qui dépassent le seul traitement de la relation marchande (comme les travaux régulationniste, conventionnaliste, old institutionalism, etc.) (cf. par exemple, KIRAT, 1993 et GILLY et PECQUEUR, 1997, sur ce point).

Les chercheurs du groupe Dynamiques de proximité font en outre de l'interaction située l'unité d'analyse essentielle (RALLET, 2002), qui permet de contextualiser l'acteur et ses relations dans son environnement. Les comportements de ce dernier doivent en effet toujours être analysés en fonction de sa place dans l'espace, et donc de sa situation par rapport à différents autres acteurs, groupes ou organisations. Ainsi conceptuellement dotée, l'approche proximiste est en mesure de s'attaquer à la compréhension du succès ou de l'échec des clusters (KIRAT et LUNG, 1999), mais aussi de s'inquiéter de la multiplication des relations à distance et de s'interroger sur leur développement et leur pérennité.

\subsection{Des enjeux empiriques et sociétaux}

D'emblée, l'Ecole de la Proximité s'est donnée pour objectif de se saisir des enjeux sociétaux et empiriques du moment. A sa création, l'après Trente Glorieuses s'amorce déjà, questionnant l'efficacité du fordisme, du toyotisme et du rapport de l'industrie aux territoires. L'Etat centralisateur s'interroge sur sa politique d'aménagement du territoire, plus «top down» que «bottom up », et sur l'utilité même d'une intervention. On annonce la " mort de la distance » (CAIRNCROSS, 1997) avec l'arrivée des «individus connectés » et des TIC qui semblent la 
supprimer, accélérant un mouvement très profond de délocalisations, même dans les industries de hautes technologies.

L'ancrage local ou territorial des firmes apparait alors comme la solution, avec la vogue des technopoles et des parcs scientifiques. La création de ressources locales et spécifiques (Colletis et PeCQueur, 2005), par nature non redéployables, empêcherait non seulement le départ des entreprises mais en attirerait de nouvelles. Les connaissances spécifiques apparaissent tout naturellement comme des exemples typiques de telles ressources du fait de leur caractère tacite, qui appelle une proximité géographique entre acteurs (ZIMMERMANN, 1998). Aux côtés de l'économie fordiste plus traditionnelle émerge progressivement une économie de la connaissance sur laquelle se fondent les pays riches pour maintenir une avance technologique : l'innovation permanente devient une nécessité, et son caractère localisé semble inéluctable.

Dès lors il s'agit de répondre à une question centrale pour les décideurs politiques,qui cèdent facilement à la mode des clusters et science parks : faut-il être proche pour innover ? Ainsi que son corollaire : de la même façon qu'il y existerait une «bonne gouvernance », existe-il une " bonne distance », « une bonne proximité »? Au-delà, même, on peut se demander si cette interrogation a du sens. D'ailleurs de quelles proximités parle-t-on? Géographiques, sociales, cognitives?

A ces questionnements, qui visent à maintenir et améliorer la compétitivité du pays, s'additionnent des préoccupations concernant la vie quotidienne des citoyens. Dès le début, de nombreux chercheurs du groupe Dynamiques de proximité se vivent comme des acteurs de la société, bien conscients de la nécessité de remplir leur devoir d'utilité sociale. Dès lors, les thématiques de déplacements pendulaires dans les villes par tous moyens de transport, en particulier, et du développement des mobilités, en général, sont naturellement mises à l'agenda du groupe (BURMEISTER et COLLETIS-WAHL, 1997). La question du travail et du salariat et leurs transformations dans un monde post-fordiste sont également à l'étude, les chercheurs cherchant à comprendre notamment quels rôles jouent les modifications du rapport salarial dans le développement territorial (PERRAT 2018 et RODET-KROICHVILI, 2018, dans ce numéro).

\subsection{Des enjeux académiques}

S'ajoutent enfin aux enjeux théoriques et «socio-empiriques" immédiats, des enjeux académiques de long terme qui visent à affirmer la place de la pensée française dans les sciences régionales européennes ainsi qu'à faire dialoguer des courants intra et interdisciplinaires. Cette approche va se révéler féconde et produire de nombreux résultats.

L'article de FILIPPI et al., 2018, dans ce numéro, qui analyse le groupe Dynamiques de proximité comme une communauté de connaissance, montre que ces enjeux sont très présents dans l'esprit des fondateurs. L'idée déjà soulignée de construire des liens forts entre économies industrielle et régionale est une façon d'occuper un espace encore vierge académiquement et ainsi d'offrir une lisibilité aux chercheurs qui ont pris le risque de quitter le mainstream, dans une période où les incitations à la publication se font plus intenses (CARRINCAZEAUX et al. 2008).

Il s'agit aussi souvent de pratiquer et de démontrer l'intérêt de l'interdisciplinarité (BELLET et al., 1998), toujours publiquement louée mais généralement académiquement sanctionnée. Là encore, on retrouve une forme assumée de prise de risque, en vue de proposer une alternative à une pensée dominante manquant de diversité, manquement dont on sait aujourd'hui qu'il est l'une des causes des lock in institutionnels. Cette ouverture intellectuelle se traduira rapidement par l'intégration de chercheurs issus d'autres disciplines, comme la sociologie ou l'aménagement du territoire, ainsi que par une capacité d'intégration de méthodes ou corpus 
analytiques provenant d'autres champs (voir récemment les emprunts à HIRSCHMAN en matière de sciences politiques par exemple).

\section{$-3-$}

\section{Les principaux résultats analytiques}

25 ans plus tard, peut-on dire que les objectifs initiaux ont été atteints ? Et si oui, dans quelle mesure ? Une autre manière de poser la question consiste à s'interroger sur les principaux résultats atteints par le programme de recherche de la proximité et à s'essayer à un premier bilan des succès, des échecs et aussi des surprises et des développements inattendus, après ces années de travail. Commençons par apporter une réponse en nous concentrant sur les enjeux théoriques, les plus importants dans le cadre du travail entrepris et de ses ambitions.

\subsection{Une réussite incontestable en matière de contributions analytiques et d'approches théoriques}

Sans conteste, une grande partie des objectifs analytiques de départ a été remplie, en particulier au niveau de la prise en compte des dimensions spatiales. L'ambition d'intégration de l'espace et des territoires dans l'étude des relations industrielles et d'innovation, ou, plus largement, au sein de l'analyse économique, partagée avec d'autres courants de pensée comme les milieux innovateurs ou les SPL (Systèmes Productifs Locaux) puis avec une partie restreinte des approches évolutionnistes, a été réalisée en plusieurs étapes, qui n'ont pas exclues les surprises ou quelques limites persistantes.

Dans un premier temps, une explication de la possible conjonction entre les approches spatiales et industrielles par incorporation et assimilation des attendus et questionnements portés par l'autre camp, a été proposée (RALLET et TORRE, 1995). Pour les analyses industrielles et de l'innovation, ce fut la démonstration qu'il n'est pas indifférent d'être situé plus ou moins près de ses concurrents, de ses fournisseurs ou des participants d'un réseau de collaboration par exemple. Pour les approches spatiales, la prise en compte des dimensions industrielles et d'innovation permettait de s'émanciper d'un tropisme souvent centré sur l'étude des modes d'aménagement ou des villes, sans réelle considération de la question entrepreneuriale. Preuve était ainsi faite que l'espace compte ( «space matters ») car il impacte de manière conséquente les relations économiques et les interactions entre acteurs dans les approches d'économie industrielle et de l'innovation. Ainsi, des firmes en situation de proximité géographique, situées dans le même périmètre, ou partageant les mêmes approches ou les mêmes ressources cognitives sont-elles davantage en mesure de collaborer ou de travailler ensemble.

Rapidement, cette idée a débordé le cadre économique productif pour s'étendre et s'imposer dans deux directions. Premièrement vers d'autres disciplines, et en particulier la sociologie et les sciences de gestion (un peu plus tard). L'analyse des réseaux d'acteurs et de leur positionnement social s'est avérée indispensable, par exemple à travers les études menées au sein des technopoles ou des firmes high tech, où les liens de connaissance, familiaux ou culturels, continuent à jouer un rôle essentiel en dépit du caractère innovant des activités (GROSSETTI et BES, 2001 ; GROSSETTI, 2008). Si l'espace est longtemps resté « un impensé de la gestion » (LAURIOL et al., 2008, p. 92), les travaux de l'école de la Proximité ont proposé une grille conceptuelle utile à la compréhension des diverses stratégies de localisation des organisations (GOMEZ et al., 2011 ; TALBOT, 2013 ; GAHINET, 2018, dans ce numéro), ainsi que des avancées par rapport à certaines approches classiques de prise en compte de l'espace en 
science régionale : l'espace comme coûts de transport, ou comme rente foncière en fonction de la distance au centre.

Deuxièmement, les développements thématiques se sont révélés tout aussi importants, la plasticité de l'approche en termes de proximités devenant évidente avec son application à des domaines de plus en plus étendus. C'est le cas des approches d'aménagement du territoire (voire les articles de NADOU et DEMAZIERE, 2018, et d'ARAGAU et al., 2018, dans ce numéro), et en particulier des procédures de négociation environnementales qui mobilisent utilement les proximités non géographiques (TORRE et BEURET, 2012). Il faut citer l'importante de la littérature sur les conflits d'usage et de l'espace, et leurs liens aux dimensions négatives et subies de la proximité géographique (MAGSI et TORRE, 2014 ; TORRE, 2011 ; voir aussi l'article de MerY et MATHIAS-MENDES, 2018, dans ce numéro). On retrouve également des analyses en termes de proximité dans les recherche sur le travail ou les rapports salariaux, ainsi qu'à la base d'une bonne partie des approches d'écologie industrielle et territoriale dans lesquelles la conjonction et la dialectique des proximités géographiques et non géographiques permet d'éclairer le lien des processus de recyclage et d'économie circulaire aux territoires où ils s'incarnent et prennent appui (BEAURAIN et BRULLOT, 2011). Plus récemment, enfin, c'est l'ouverture aux problématiques de développement territorial qui marque une nouvelle extension du champ de l'analyse.

Il en découle une clarification d'ensemble des liens entre les concepts d'espace et de proximité dans leurs différentes déclinaisons. Par exemple, alors que proximité et localisation sont fréquemment confondues (TORRE et RALLET, 2005), on montre que la proximité est plurifactorielle et s'abolit souvent des localisations: on peut être proche à distance, par l'intermédiaire des communications, ou rester proche de sa famille habitant dans un autre pays. L'espace n'est donc pas un simple support, mais un assemblage de ressources, auquel les proximités donnent accès (COLLETIS et PECQUEUR, 2005 ; ZIMMERMANN, 1998) Par ailleurs, il est également un vecteur de leur processus de construction voire de spécification. En partie créé par les acteurs, sa maitrise ainsi que sa puissance de contrainte sur ces derniers dépendent largement du jeu des proximités ainsi que de leurs mobilisations par des groupes d'acteurs certes situés, mais qui agissent localement ou à distance. Une vraie complexité des rapports s'installe ainsi, qui nécessite une analyse fine et une batterie de concepts et d'indicateurs sans cesse plus sophistiqués.

Cette conception repensée du lien entre espace et proximité, qui s'impose en économie spatiale et dans les sciences régionales, ainsi que l'intégration d'éléments provenant des approches organisationnelles et institutionnelles, ont permis l'ouverture de la boite noire des externalités de proximité. Alors que les premiers travaux posaient comme un challenge la compréhension des relations de proximités au sein des systèmes productifs par exemple, le chercheur qui désire aujourd'hui étudier des relations interentreprises, mais aussi tout autre type d'interactions entre différentes catégories d'acteurs au niveau local, dispose d'un ensemble théorique et analytique qui lui permet d'utiliser les outils de la proximité comme autant de clés de lecture et de compréhension du réel.

Elle a également ouvert la porte à des apports importants sur la question territoriale. Les recherches sur les relations firmes-territoires ont montré que ces derniers ne sont pas des entités closes. Puisque les acteurs économiques sont parties prenantes de circuits qui dépassent leur localisation, les territoires ne peuvent prétendre se développer sur des principes autarciques mais ont besoin d'articulations externes pour assurer l'accès à des intrants et à des marchés, et pour rester en connexion avec des dynamiques externes, technologiques, organisationnelles, voire sociales. Ces connexions/coordinations externes reposent sur des proximités nongéographiques ou organisées, maîtrisées en général par un nombre réduit d'agents, publics ou 
privés qui jouent un rôle de gatekeepers, assurant l'articulation entre des ensembles locaux, et faisant système autour de l'activation de proximités géographiques avec des entités ou des circuits globaux (RYCHEN et ZIMMERMANN, 2008). Du coup, le territoire est envisagé comme un construit, susceptible d'effondrement, de fractionnement ou d'éclatement, mais aussi de reconfiguration de résurgence et de renouveau selon les proximités qui pourront y être activées (TORRE et BEURET, 2012). Ceci questionne bien entendu l'action publique, trop souvent limitée à des zones sur lesquelles s'exercent les prérogatives des collectivités publiques. Les proximités ne peuvent s'enfermer dans ces découpages administratifs et doivent s'en affranchir pour pouvoir se déployer en termes de systèmes économiques et faire la part belle aux initiatives des acteurs locaux.

Il en résulte une variété de définitions des dimensions de la proximité, qui n'est pas un désordre théorique mais bien le fondement d'une heuristique capable de capter la complexité du monde social et de ses relations à l'espace. Certes, les définitions ont fleuri, et la tentation d'ajouter une proximité de plus à une liste déjà longue est souvent irrésistible. Mais, au cours du temps, deux grandes familles ont émergé et se sont imposées dans la littérature depuis une dizaine d'années. D'une part, la définition des 5 grandes catégories de proximités définies par Boschma (2005), pour l'Ecole Hollandaise, et qui constitue la base de l'essentiel des recherches appliquées, de loin les plus nombreuses, menées dans le domaine des relations d'innovation et de technologie. De l'autre, celle retenue par différents auteurs (en particulier TORRE et RALlet, 2005, et PECQueur et ZimMERMANn, 2004), qui domine largement dans le monde francophone, avec deux grandes proximités, géographiques et organisées, elles-mêmes divisées en sous catégories.

Ces déclinaisons de proximités se combinent ou s'opposent plus ou moins harmonieusement selon les actions des acteurs humains, qu'ils agissent en groupe ou à titre individuel. En dépit de leur apparente diversité, elles se fondent toutes sur un principe de franchissement des distances de différentes natures (spatiales ou non) entre êtres humains, ou avec des acteurs non humains (être vivants ou lieux). L'action des individus, par le biais de la mobilisation des proximités, vise à résoudre la question, en tentant de recréer ou de briser le lien qui s'impose ainsi. Par exemple un système local de production ne sera efficace que par la combinaison de relations de proximités géographiques et organisées, alors qu'un conflit de voisinage provoqué par la proximité subie de voisins pourra trouver un certain apaisement par mobilisation de liens de proximités organisées. Ce que l'on a tendance à appeler aujourd'hui la «grammaire des proximités » offre ainsi une capacité d'explication importante des problématiques spatiales par mobilisation de ses différentes déclinaisons. Ce pouvoir explicatif provient de la capacité d'un cadre conceptuel devenu « agile » à tenir compte de la complexité et de la diversité du réel, à s'adapter aux faits sans les dénaturer. Mais cette agilité semble toutefois avoir un prix.

\subsection{Des limites et des échecs}

A côté de ces succès, il faut en effet pointer quelques limites ou échecs récurrents, qui correspondent à des objectifs non atteints, bien qu'identifiés dans l'agenda de départ. Ils concernent en particulier la définition d'une formalisation et d'une mesure solide des proximités, qui ne semble pas prête d'émerger en dépit de tentatives allant dans ce sens (BOUBA OlgA et ZiMMERMANN, 2004). Ce point pose question car il interroge la possibilité d'aboutir à une modélisation précise et de la fixation définitive des contours de la notion de proximité, ainsi que de son statut analytique : théorie ou heuristique ? Au vu du développement des instruments de mesure empirique des proximités de toutes natures - mesures de distances géographiques de différentes sortes, corrélations économétriques, analyse des réseaux sociaux, analyse formalisée 
des discours, cartes mentales... - il semble plus raisonnable de tabler sur la possibilité de rendre compte de la variété et de la multiplicité des formes prises par les proximités dans la réalité, et d'en faire éventuellement usage pour des interventions dans le cadre de l'action publique, plutôt que d'attendre une hypothétique définition canonique de la proximité. Elle supposerait de mettre en œuvre un arsenal conceptuel centré à la fois sur l'espace, la distance et le lien social, qui ne garantirait pas l'obtention d'une telle définition.

Un autre échec historique concerne l'incapacité à s'articuler avec les programmes de recherche d'autres approches pointées à l'origine comme voisines ou nourricières, et en particulier les analyses régulationnistes et conventionnalistes de l'économie. L'article de DORE, 2018, dans ce numéro, propose d'ailleurs une analyse précise des tentatives plus ou moins réussies d'introduction des travaux régulationnistes et conventionnalistes dans la pensée de l'Ecole de la proximité. Au contraire, cet objectif semble s'être éloigné et la disjonction ou le désintérêt deviennent parfois forts. A cela deux raisons : d'une part l'élargissement disciplinaire des approches de la proximité au-delà du seul cadre de l'économie et les emprunts ainsi faits à d'autres sciences, indifférentes à ces débats ; d'autre part des tiraillements internes assez forts, avec en particulier le développement de nombreuses approches plus mainstream de la proximité, chemin faisant avec les analyses interactionnistes, l'économétrie des variables qualitatives, l'étude des volumes de R\&D ou de la course aux brevets, l'étude des réseaux sociaux...

\subsection{Des réussites et extensions inattendus}

On constate enfin que de nombreux développements importants des analyses de la proximité ne correspondent pas à des objectifs fixés dans l'agenda de départ. Cela n'a sans doute rien d'étonnant pour une approche qui se déploie sur une période de 25 ans, mais traduit la vitalité et la plasticité du programme de recherche et des choix analytiques opérés. Retenons trois illustrations emblématiques de ces évolutions.

Tout d'abord, les dimensions temporaires de la proximité géographique. Il est apparu, après une dizaine d'années, que limiter la proximité géographique à un cadre permanent, que l'on trouve dans les approches des clusters ou de l'ancrage des firmes par exemple, ne suffit pas à expliquer les évolutions contemporaines des relations entre acteurs industriels et de l'innovation, et audelà. En effet, le développement des technologies de l'information et de la communication (TICs) a permis la naissance de relations à distance, en particulier par le biais des terminaux ou des réseaux sociaux, qui signent à la fois une forte remise en cause de l'importance de la géographie et l'apparition d'individus doués du don d'ubiquité pouvant éventuellement agir à deux endroit en même temps (RALLET, 2002 ; TORRE, 2008). Mais loin d'en conclure à la mort de la distance, les approches de la proximité, s'appuyant sur de nombreuses études empiriques, ont montré que la proximité géographique continue à compter, mais souvent de manière temporaire (TORRE, 2010). C'est le cas des firmes engagées dans des programmes de collaboration, qui doivent se réunir au début du projet puis de manière régulière pour fixer les éléments de travail, élaborer la confiance et rapprocher les cartes cognitives (à l'instar des équipes plateaux dans l'industrie aéronautique, GILLY et al., 2011) mais aussi pour les chercheurs qui se retrouvent dans des colloques ou des foires, ainsi que pour les membres de diasporas se retrouvant à intervalles réguliers pour reconfirmer leurs liens d'appartenance (TORRE, 2011).

Ensuite, la caractérisation des proximités selon qu'elles sont souhaitées ou subies. Les premières approches s'intéressaient avant tout à des processus ou stratégies de recherche de proximités, géographiques ou non spatiales, par exemple par des firmes cherchant à se localiser 
à faible distance les unes des autres, ou par la construction de proximités organisées au sein de réseaux de collaboration ou de co-construction de connaissances (voir JACQUIER-ROUX, 2018, dans ce numéro). Mais, avec l'élargissement à d'autres types d'acteurs et de situations est apparue l'idée que les proximités pouvaient présenter des dimensions plus négatives ou être subies (TORRE et BEURET, 2012). A commencer par les approches des conflits, qui reposent sur la constatation que des acteurs subissent la proximité géographique d'autres acteurs ou de leurs activités, génératrices de nuisances telles que le bruit, la pollution, l'occupation des sols, la destruction des paysages... . Le parallèle avec les proximités organisées s'est ensuite imposé, avec les inconvénients de type imitation ou espionnage industriel causés par les excès de ressemblances en termes de technologies entre firmes d'un même réseau, ou l'incapacité à différencier les productions de firmes concurrentes.

Enfin, les proximités permettent tout autant un partage (par exemple de connaissance) qu'une régulation des relations (LEVY et TALBOT, 2015). De nombreuses relations économiques ne sont pérennes que parce qu'elles autorisent un échange qu'il est nécessaire de contrôler. Si les proximités entre acteurs favorisent le partage (une proximité géographique favorise le partage de connaissances tacites via le face à face par exemple), elles sont aussi l'occasion de contrôler les conditions et les résultats du dit partage effectué (le face à face offrant un accès rapide aux informations en fluidifiant les relations tout en réduisant l'incertitude et les risques d'opportunisme (voir TALBOT, 2018, dans ce numéro, pour une proposition d'un cadre théorique qui analyse les effets de contrôle des proximités). Finalement, c'est une façon de réintroduire dans l'analyse des proximités les effets du pouvoir, ce «banni récalcitrant » cher à François PERROUX.

\section{$-4-$}

\section{Les acquis académiques et sociétaux}

L'ambition de départ du groupe Dynamiques de Proximité était avant tout de nature analytique et théorique, et présentait donc des enjeux académiques, à la croisée des disciplines et des programmes de recherche. Ancrées dans le réel, ces recherches s'avéraient également porteuses d'enjeux de société, qu'elles prétendent décrire la réalité des relations économiques et sociales ou désirent apporter des recommandations en matière d'actions ou de politiques publiques. Essayons d'évaluer et de préciser la réussite de ce programme, ainsi que son impact, dans les paragraphes qui suivent.

\subsection{La proximité au cœur des interactions avec la société}

Nées dans un contexte particulier de découvertes des dimensions spatiales de l'innovation et de fin du fordisme triomphant et a-territorial piloté par des multinationales hors sol, les approches de la proximité renvoyaient à une prise en considération, souvent confuse, de valeurs spatiales et territoriales, qui n'a fait que s'affirmer depuis avec l'apparition de de nouvelles problématiques concernant la traçabilité des produits, les circuits courts, les tiers lieux, les open lab, l'analyse des politiques publiques locales, la e-santé et le parcours patient... Il n'est pas très étonnant que le succès sociétal ait été au rendez-vous, et que l'expression même ait provoqué un vif engouement, loin d'être attribuable au seul groupe Dynamiques de proximité bien sûr. On parle de justice de proximité, de police de proximité, de magasins de proximité..., et les hommes politiques se veulent proches de leurs concitoyens. 
Au-delà de cette reconnaissance un peu artificielle, c'est avant tout la possibilité de faire usage des différentes proximités comme des composantes d'une boite à outils dans le cadre d'actions ou de politique publiques qui doit attirer l'attention. A partir du moment où leurs potentiels sont mis en évidence, il apparait que ces proximités peuvent être mobilisées et utilisées comme un trousseau de clés permettant d'instrumentaliser l'action publique. Les pouvoirs publics vont agir pour renforcer les proximités dans le but de légitimer et de renforcer l'efficacité de leur action. Mais, dans le même temps, des acteurs des territoires mobilisent les proximités pour se constituer en communautés, en groupes de contestation ou en réseaux d'expérimentation sociale. Par exemple, l'organisation de groupes locaux de citoyens dans le cas d'oppositions ou de conflits virulents, ou l'installation de tiers lieux dans des zones en déficit de lien social, la mise en place de moyens de transport ou de lieux dédiés dans des territoires isolés ou en difficultés de transports publics, etc. apparaissent comme des solutions mises en œuvre par les acteurs publics ou par des acteurs des territoires et faisant appel aux effets positifs des proximités.

On comprend alors que le concept de proximité voisine et cousine avec d'autres termes apparus dans le débat public comme les mobilités de toutes natures, le numérique et les TICs, les circuits courts... sans se confondre pour autant avec ces derniers. Sans entrer dans des débats oiseux, la robustesse de l'approche analytique permet d'éviter certains pièges : les proximités ne sont pas toujours positives, elles peuvent avoir des influences diverses sur les individus ou la société, elles ne s'imposent pas mais doivent être construites et mobilisées par les acteurs... En somme, une subtilité qui vient contrecarrer l'idée simple suivant laquelle la proximité (géographique) est bonne pour les firmes d'un cluster et donc qu'il faut favoriser la concentration spatiale d'entreprises.

\subsection{Des objectifs académiques atteints ou dépassés}

On peut affirmer sans peine que l'objectif académique de départ a été atteint, voire largement dépassé. Les analyses de la proximité font aujourd'hui partie de la boite à outils de la Science Régionale, qu'elles ont contribué à fortement élargir, et ont tout à fait perdu le caractère hétérodoxe ou exotique des premières tentatives, comme le prouve le nombre impressionnant d'articles publiés sur la question et de travaux utilisant les méthodologies proposées, ainsi que les ouvrages et manuels qui y font référence comme à une approche reconnue, voire banalisée maintenant.

Le succès ne s'est pas limité à la France, grâce une internationalisation réussie, qui a reposée sur les efforts des membres du groupe, mais avant tout sur la reprise des éléments de base de l'approche et la définition de catégories très opérationnelles et souvent mesurables par BOSCHMA, puis par d'autres chercheurs non francophones après lui (citons, de façon non exhaustive, Boschma et IAmmarino, 2009 ; Boschma et Frenken, 2010 ; Basile et AL., 2012). Aujourd'hui coexistent deux grandes écoles de la proximité, qui intègrent les dimensions industrielles et spatiales, dans des proportions parfois très variables selon les auteurs, mais vont bien au-delà aux niveaux disciplinaires et thématiques. L'école hollandaise, largement liée à la géographie économique évolutionniste, se distingue par sa capacité à mesurer différents types de proximités, le plus souvent non spatiales, la mise en évidence du principe de variété reliée et des études ciblées sur les relations industrielles et d'innovation, souvent au sein de systèmes localisés (voir l'article de BRUNELLE et DUBE, 2018, dans ce numéro). L'école française est plus diversifiée car elle s'est développée en paliers successifs, d'abord autour des relations industrielles et d'innovation et tout en explorant différentes architectures de proximités, puis en s'ouvrant à des approches pluridisciplinaires et à des sujets nouveaux comme l'environnement 
(TORRE et ZUINDEAU, 2009) ou l'aménagement, puis en inférant une grammaire élargie qui intègre des déclinaisons comme les proximités temporaires ou recherchées/subies.

C'est l'occasion d'affirmer internationalement une sensibilité francophone en matière de sciences régionales et/ou de sciences du territoire et d'inscrire ces recherches dans la tradition de la recherche française en sciences sociales. Le travail se situe dans la filiation des grands auteurs en économie régionale comme Perroux, Boudeville, Aydalot, Maillat..., avec lesquels un subtil ballet d'attirance et de répulsion va se dérouler, y compris en termes de rejet ou d'adhésion aux institutions académiques. Mais une tradition plus large des sciences sociales est également convoquée. BOURDIEU (avec la reproduction et la définition des relations sociales et des séparations qu'elles imposent), FOUCAULT (avec les dispositifs de gouvernance et leur déclinaison territoriale ou les hétérotopies), DELEUzE (avec la dimension ryzomique des réseaux et la déterritorialisation), MORIN (avec la complexité et le caractère systémique des relations), souvent cités et aux influences revendiquées, voisinent avec d'autres références étrangères comme celles de GiDDENS, VEBLEN, WHITE ou HIRSCHMAN.

\section{$-5-$}

\section{Les angles morts, le travail restant à réaliser}

Aujourd'hui, pour l'anniversaire des 25 ans du programme de recherche sur les proximités, la question se pose : comment prolonger le travail et les analyses, et surtout quelles sont les questions qui n'ont pas encore été couvertes et réclament un traitement par les approches de la proximité, voire quelles sont les dimensions de la proximité qui n'ont pas encore été explorées et mériteraient que l'on s'y attache ou que l'on s'y attaque maintenant?

\subsection{Définitions et mesures de la proximité}

Une première voie de recherche concerne la définition de la proximité elle-même, qui n'est jamais caractérisée autrement qu'à travers ses diverses dimensions. Signe d'immaturité d'une approche encore jeune ? La proximité est sûrement une heuristique qui a fait ses preuves, un outil ouvrant la voie à la découverte scientifique dont nous venons de rappeler les résultats théoriques, socio-empiriques et académiques.

Une façon immédiate d'aborder cette difficile question est de différencier la proximité d'autres concepts. Une partie du chemin a d'ores et déjà été accomplie : comme souligné plus haut, la distinction entre proximité et espace ou localisation est opérée. Mais la démarche peut encore être poursuivie. L'espace offreur de ressources accessibles via la proximité peut par exemple être saisi comme une production sociale au sens de LEFEBVRE (1974). Il devient manipulable par certains acteurs dans le sens de leurs intérêts et peut être utilisé pour exercer un pouvoir : l'espace est un outil de domination des détenteurs du pouvoir, ou un dispositif de résistance mobilisé par les acteurs qui subissent ce même pouvoir (TAYLOR et SPICER, 2007). Cette conception ouvre de nouvelles perspectives sur les effets de proximité. C'est aussi une façon de redynamiser une lecture institutionnaliste des rapports de proximité en introduisant frontalement la question du pouvoir. Dans une perspective plus interindividuelle cette fois, l'espace permet d'induire des comportements par les proximités créées : l'architecture d'un bâtiment oriente ainsi les déplacements de ses occupants, en créant par exemple des lieux de rencontre, tandis que les open space, s'ils visent à favoriser les échanges spontanés entre les individus, les incitent aussi à s'autoréguler puisqu'ils travaillent au vue de tous : les face à face 
permanents provoquent ici des comportements d'échanges et d'autocontrôle et modifient bien entendu les proximité organisées.

De fait, la question de la proximité (des proximités) et de son rapport à la distance (aux distances) n'est pas vraiment élucidée. Certes, il est depuis longtemps acquis que les distances en question ne sont pas seulement spatiales, ou géographiques, et que même quand c'est le cas elles s'assortissent d'un certain nombre de conditions sociales comme les moyens d'accès ou le prix. Il est clair également que si les distances existent toujours, ce n'est pas le cas des proximités. Ces dernières sont en effet latentes et doivent être activées pour prendre consistance et devenir éventuellement des outils au service de l'action. Il s'agit de réponses construites par les acteurs au problème de l'éloignement et de son comblement, par mobilisation de leurs caractéristiques géographiques ou sociales. Plusieurs questions restent alors non résolues. Par exemple, comment se réalise le franchissement de cette distance ? Entre la coupure et la frontière, s'agit-il d'un continuum, ou existe-t-il des phénomènes de rupture ? (la question doit être posée aux niveaux spatial et non spatial). Est-il possible de sortir d'une logique binaire des proximités (je suis proche de, ou pas proche de), et d'imaginer des degrés de proximités, comme il y a des échelles de distance, ou est-ce que cela n'a aucun sens?

En fait, les approches de la proximité n'ont pas signé la mort de la distance mais elles l'ont salement amochée quand même, en expliquant pourquoi on pouvait réaliser un grand nombre d'activités en dehors de la coprésence. La proximité géographique temporaire (TORRE, 2008), caractéristique de l'époque post-moderne, est-elle en mesure de résister au développement du numérique, aux progrès de la réalité virtuelle et à la montée des big data? Par ailleurs, comment s'accommoder du fait que les proximités, en raison de leur caractère social, incorporent une part importante de perception. Comment expliquer des perceptions différenciées des proximités par des acteurs interagissant pourtant ensemble : l'un se sent proche, l'autre loin ; l'un souhaite la proximité, l'autre la subit. Comment intégrer cette dimension essentielle dans l'approche et la grammaire même des proximités ? Par une lecture en terme de rapports de pouvoir, comme rappelé plus haut, mais est-ce la seule approche possible ? Et comment la rendre compatible avec certaines formes de modélisation ou de mesure?

La question récurrente de la mesure demeure ainsi toujours d'actualité. D'autant que nous savons que la proximité, fait largement social, ne s'accorde pas avec l'idée d'optimalité, ce qui en faciliterait la quantification. Il ne faut pas en effet confondre le paradoxe apparent de la proximité - qui veut que trop près, on subit des effets négatifs (entassement spatial, manque de complémentarité de connaissances trop similaires...) et que trop loin on ne peut pas interagir car la distance devient infranchissable - avec l'idée fausse qu'il y aurait une proximité optimale, et donc idéale. Ce piège évité, des progrès ont été accomplis dans l'idée d'une quantification objective des proximités en utilisant des méthodologies issues de l'analyse des réseaux (VICENTE et al., 2018, dans ce numéro), des échelles de mesures (GELDES et al., 2015), de la comparaison des classes internationales des brevets (BOSCHMA et IAMMARINO, 2009), des analyses des correspondances multiples et autres classification ascendante hiérarchique (LETHIAIS, 2018, dans ce numéro), etc. Mais beaucoup reste à faire.

Une façon de contourner le problème est peut-être de mesurer les effets des proximités et non ces dernières directement, et de recourir à des concepts comme la densité, pour étalonner les possibilités de connexion, et la diversité qui apporte de la richesse à la qualité des interactions. Cela sans verser dans une trop forte dérive objectiviste, soulignée par AGUILERA et al. (2015), qui conduit à délaisser toute idée d'évaluation de la dimension subjective des proximités audelà des seules études de cas. C'est peut-être à ce niveau que des progrès plus rapides sont à espérer, la confrontation à cet enjeu appelant le développement des méthodes basées sur les 
analyses de contenu thématique d'entretiens (HAMOUDA et TALBOT, 2018) ou les cartes mentales (CARIOU et al., 2018, dans ce numéro) par exemple.

\subsection{Dynamiques et dimension géographique}

Une autre limite, presque un paradoxe au regard du nom du groupe, consiste dans la prise en compte limitée des processus dynamiques de proximité. Certes, cette dimension n'est pas absente de l'analyse et aucun auteur proximiste ne prétendra que la question de la dynamique ne soit pas importante, ni que l'approche proposée ne puisse avoir des conséquences en termes de dynamiques, et plusieurs se sont clairement inscrits dans une approche évolutionniste. Mais force est de constater que les modélisations (au sens large) dynamiques existent peu. La prise en compte des effets successifs des dynamiques de proximités sur leur environnement, ou des impacts de ce dernier sur les proximités à moyen et long terme et des transformations ainsi subies, ainsi que l'influence croisée des proximités dans le temps ne sont que rarement envisagées. Deux exceptions à cela toutefois. L'approche de BALLAND et al (2015), qui propose un modèle de dynamique centré sur la coévolution des réseaux de connaissances pour innover et de différentes formes de proximités. Et la modélisation des séquences de proximités géographiques temporaire ou permanente et de proximités organisées présentée par TORRE (2010) (voir à ce sujet la reformulation et l'extension de ce modèle proposées par GALLAUD, 2018, dans ce numéro). Toutefois, beaucoup reste encore à faire pour démêler l'écheveau des influences réciproques et des effets croisés des proximités, ainsi que de leur impact dans le temps.

Enfin, on ne peut qu'être frappé par une certaine légèreté générale sur la question de la proximité géographique, trop souvent perdue de vue dans l'analyse. On constate en effet une tendance massive à un oubli de cette dimension, souvent revendiquée mais aussi généralement négligée dans l'analyse alors que sa dialectique avec les autres formes de proximités (organisées) est à la base même des approches de la proximité (TORRE, 2009 ; TALANDIER et PECQUeur, 2018). Il est paradoxal qu'un programme de recherche qui s'est construit sur l'intégration de l'espace dans l'approche économique en vienne à minimiser la place de la variable spatiale. D'aucuns pourraient simplement y voir un retour des forces favorables à une séparation des disciplines et à une prise en compte minimaliste de l'espace, sous ses formes les plus proches de l'espace point ou de la donnée mathématique. Il faut également sans doute pointer ici la difficulté de contact avec la géographie, par des jeux de concurrence académique qui ont empêché la réelle émergence de travaux communs (PUMAIN, 2006).

De grandes possibilités restent pourtant ouvertes à l'analyse dans ce domaine, en particulier au niveau de l'approche des territoires, un peu négligée pendant un temps mais qui revient maintenant en force avec l'intégration des dimensions de développement territorial (voir l'article de TORRe, 2018, dans ce numéro). Se poser des questions de développement, de gouvernance des territoires, mais également de construction, de mobilisation et de révélation des ressources locales revient à mettre la proximité géographique au cœur de l'analyse, à lui redonner un rôle clé et à s'interroger à la fois sur son rôle, ses caractéristiques, ses fonctions, ses limites et ses possibilités d'activation dans le cadre des dialectiques complexes de localisation et de globalisation (voir l'article de COLLETIS et PECQUEUR, 2018, dans ce numéro). 


\section{Conclusion}

Les approches de la proximité peuvent-elles encore se développer et prendre de l'ampleur ou sont-elles maintenant condamnées à un ronronnement institutionnel? Et surtout, sont-elles encore en mesure de conserver un caractère innovant? Comment lutter contre l'institutionnalisation et la perte de fraicheur qui l'accompagne? La question reste posée, et si l'on ne peut évidemment faire de pronostics raisonnables, il n'en demeure pas moins que cette possibilité dépend de nombreux facteurs, de différentes natures. Essayons de l'examiner à l'aune de nos trois grandes catégories, analytiques, académiques et sociétales.

D'un point de vue analytique des champs s'ouvrent encore, comme indiqué précédemment, dans différentes directions. Il s'agit en particulier des modalités de dynamisation des proximités et de la manière dont elles interagissent et interfèrent les unes avec les autres dans le temps : quel est l'impact d'une mutation des relations de proximité géographique sur l'organisation des échanges ou des interactions par exemple, ou quelles sont les conséquences de la multiplication des interactions à distance sur les modalités d'organisation de la production et de l'occupation de l'espace sur un territoire? Mais aussi, du rôle de l'espace et des territoires, où le programme de départ n'a été qu'imparfaitement réalisé, probablement par manque d'intérêt et d'éclairage des spécialistes en géographie en particulier. La question de la proximité géographique reste encore largement une énigme : est-ce une banalité ou l'expression d'un apport social complexe à l'espace ?

D'un point de vue académique, il s'agit essentiellement de l'extension à de nouveaux champs ou de nouvelles disciplines. A l'heure actuelle, ce sont les sciences de gestion qui manifestent le plus grand intérêt, sans doute parce que cette discipline, friande de cadres conceptuels nouveaux, a besoin de comprendre le rôle de l'espace dans le fonctionnement des organisations, alors même que les théories des organisations sur laquelle elle s'appuie traditionnellement sont muettes sur ce point. Elles peuvent à leur tour contribuer au développement de l'Ecole de la Proximité en proposant des avancées théoriques et méthodologiques. D'un point de vue thématique, c'est avant tout l'écologie industrielle et territoriale ou l'économie circulaire qui doivent attirer l'attention, car elles combinent les dimensions d'ancrage territorial avec les problématiques technologiques et la mobilisation de réseaux d'acteurs.

Enfin, d'un point de vue sociétal, l'intérêt pour les proximités ne se dément pas dans le débat public et le terme reste indéniablement à la fois terriblement vague et facteur de modernité et de participation. Reste toutefois à lui donner un contenu plus ferme en termes d'action publique et à définir ce que pourraient être des actions ou politiques locales de proximité qui ne se limitent pas à une déclinaison des avantages de la proximité géographique ou des relations de voisinage, mais intègrent les modalités de mobilisation des différents types de proximités et leurs interférences dans un cadre territorial ouvert aux courants de la globalisation.

\section{Remerciements}

Les auteurs tiennent à remercier Maryline Filippi et Frédéric Wallet pour leur relecture attentive et constructive de cette introduction. Nous restons totalement responsables des imperfections et toujours possibles erreurs que pourraient comporter ce travail. 


\section{Références bibliographiques}

Aguilera A, Lethiais V, Rallet A, (2015) Spatial Proximity and Intercompany Communication: Myths and Realities. European Planning Studies, 23(4): 798-810.

Aragau C, Bouleau M, Mangeney C (2018) Les bassins de vie ont-ils un sens ? Une interrogation de la proximité en périurbain francilien. Revue d'Économie Régionale et Urbaine, 5-6 : XXXX

Balland P-A, Boschma R, Frenken K (2015) Proximity and Innovation: From Statics to Dynamics. Regional Studies 49(6): 907-920.

Basile R, Capello R, Caragliu A (2012). Technological interdependence and regional growth in Europe: Proximity and synergy in knowledge spillovers, Papers in Regional Science, 91, 4, 697-880.

BeAurain C., BRULlot S., 2011. L'écologie industrielle comme processus de développement territoriale : une lecture par la proximité, Revue d'Économie Régionale et Urbaine, 2, 313-340.

Bellet M, Colletis G, Lung Y (dir.) (1993) Economie de proximités. Revue d'Economie Régionale et Urbaine $\mathrm{n}^{\circ} 3$.

Bellet M, KiRAT T (1998) La proximité, entre espace et coordination. In : BELleT M, KIRAT T, LARGERON C (dir.) Approches multiformes de la proximité. Hermès, Paris.

Bellet M., Kirat T., LARGERON C. (Coord.) (1998), Approches multiformes de la proximité, Hermès, Paris.

Boschma R (2005) Proximity and innovation. A critical assessment. Regional Studies 39(1): 61-74.

BOSCHMA R, IAMMARINO S (2009) Related variety, trade linkages, and regional growth in Italy, Economic Geography, 85(3):289-311.

BOSCHMA R, FRENKEN K (2010) The spatial evolution of innovation networks: a proximity perspective. In: BOSCHMA R, MARTIN R (eds) The Handbook of Evolutionary Economic Geography. Edward Elgar: Cheltenham, Northampton.

Bouba-Olga O, CARrincAzeAux C, CORIS M (dir) (2008) La proximité : 15 ans déjà ! Revue d'Economie Régionale et Urbaine, $\mathrm{n}^{\circ} 3$.

Bouba Olga O, Zimmermann J-B (2004) Modèles et Mesures de la Proximité » in Pecqueur B. et Zimmermann J.B. (Eds.), Economie de Proximités, Hermès.

Burmeister A, Colletis-Wahl K, (1997) Proximity in Production Networks. The Circulatory Dimension. European Planning Studies, 4(3): 231-241.

BRUNELle C, DubE J, (2018) De l'importance de la proximité dans la capacité de résister aux chocs exogènes. Une analyse de survie d'entreprise durant la Grande Récession dans la région périphérique du Bas-Saint-Laurent, Canada. Revue d'Économie Régionale et Urbaine, 5-6: XXXX

Cariou C, Ferru M, Rallet A (2018) Perceptions des lieux et proximités subjectives : une analyse des dynamiques créatives franciliennes. Revue d'Économie Régionale et Urbaine, 5-6 : XXXX

CaIrncross F. (1997) The Death of Distance: How the Communications Revolution Will Change Our Lives, Harvard Business School Press, 303 p.

CARRINCAZEAUX C, LUNG Y, ViCENTE J (2008) The scientific trajectory of the French school of proximity: interaction- and institution- based approaches to Regional System of Innovation. European Planning Studies, 16(5): 617-628.

Colletis G, PeCQueur B (2005) Révélation de ressources spécifiques et coordination située. Economie et Institutions, 6-7 : 51-74. 
Colletis G, Pecqueur B (2018) Révélation des ressources spécifiques territoriales et inégalités de développement. Le rôle de la proximité géographique. Revue d'Économie Régionale et Urbaine, 5-6 : XXXX

DORE G (2018) La proximité au prisme du débat entre «conventionnalistes » et « régulationnistes ». Un institutionnalisme sans institutions? Revue d'Économie Régionale et Urbaine, 5-6 : XXXX

DupuY C, GILly J-P (1995) Les stratégies territoriales des grands groupes. In : RALLET A, TORRE A (dir.) Economie industrielle et économie spatiale. Economica, Paris.

DupUy C., BurmeISTER A. (Eds.) (2003), Entreprises et territoires. Les nouveaux enjeux de la proximité, La Documentation française, Paris.

Dupuy C, Torre A (2004) Confiance et proximité. In : PeCQueur B, Zimmermann J-B (dir.) Economie de Proximités. Hermès, Paris.

Filippi M, Wallet F, Polge E (2018) L'école de la Proximité : Naissance et Evolution d'une Communauté de Connaissance. Revue d'Économie Régionale et Urbaine, 5-6 : XXXX

GAHINET M-C (2018) Les dimensions de la proximité appliquées aux achats alimentaires. Revue d'Économie Régionale et Urbaine, 5-6 : XXXX

GALlaud D (2018) Dynamiques de proximité : pistes de réflexion pour un enrichissement des modèles. Le cas de l'approvisionnement de la restauration collective. Revue d'Économie Régionale et Urbaine, 5-6 : XXXX

Gilly J-P, PeCQueur B (1997). Régulation et territoire, une approche des dynamiques institutionnelles locales. Deuxièmes Journées de la Proximité, Proximités et coordination économique, Lyon, mai.

Gilly J-P, TALBOt D, Zuliani JM (2011), Hub firms and the dynamics of regional innovation: case studies of Thales and Liebherr in Toulouse. European Planning Studies, 19(12) : 20092024.

Gilly J-P, TORRe A. (Dir.) (2000), Dynamiques de proximité, L'Harmattan, Paris.

Gomez PY, Rousseau A, VAndangeon-Derumez (2011) Distance et proximité : esquisse d'une problématique pour les organisations. Revue Française de Gestion, 4(213) :13-24.

Grossetti M (2008) Proximities and embedding effects. European Planning Studies 16(5): 629-642.

Grossetti M, Bes M-P (2001) Interacting individuals and organizations: a case study on cooperations between firms and research laboratories. In : KIRMAN A, ZIMMERMANN J-B (dir.), Economics with heterogeneous interacting agents. Springer, Berlin.

Geldes C, Felzensztein C, Turkina E, Durand A (2015). How does proximity affect interfirm marketing cooperation? A study of an agribusiness cluster Journal of Business Research $68: 263-272$.

Hamouda I, TALBOT D (2018) Contenu et effets de proximité institutionnelle : un cas d'enfermement dans l'industrie aéronautique. Management et Avenir, 3(101) : 105-129.

JACQUIER-ROUX V (2018) Vers une déconcentration géographique de la production des connaissances ? Mobilité des chercheurs et coopérations dans les réseaux de connaissances. Revue d'Économie Régionale et Urbaine, 5-6 : XXXX

KIRAT T (1993) Innovation technologique et apprentissage institutionnel : institutions et proximité dans la dynamique des systèmes d'innovation territorialisés. Revue d'Economie Régionale et Urbaine, 3 : 547-564.

KIRAT T, LUNG Y (1999) Innovation and proximity-Territories as loci of collective learning processes. European Urban and Regional Studies 6(1): 27-38. 
KRUGMAN P (1995) Rendements croissants et géographie économique. In : RALLET A, TORRE A (dir.) Economie industrielle et économie spatiale. Economica, Paris.

LAURIOL J, PERRET V, TANNERY F (2008) Stratégies, espaces et territoires. Une introduction sous le prisme géographique. Revue française de gestion, 4(184) : 91-103.

LEFEBVRE H (1974) La production de l'espace. L'Homme et la société, 31-32 : 15-32.

LETHIAIS V (2018) Proximités, coopération et innovation : que nous apprennent les travaux empiriques menés sur les PME ? Revue d'Économie Régionale et Urbaine, 5-6 : XXXX

LEVY R, TALBOT D (2015) Control by proximity: Evidence from the "Aerospace Valley" competitiveness cluster. Regional Studies 49(6): 955-972.

Magsi H, TORRe (2014) Proximity analysis of inefficient practices and socio-spatial negligence: Evidence, evaluations and recommendations drawn from the construction of Chotiari reservoir in Pakistan, Land Use Policy, 36, 567 - 576.

Mery J, MATIAS-Mendes M (2018) Une analyse des installations localement indésirables en termes d'économie de la proximité : application à la gouvernance territoriale du stockage des déchets. Revue d'Économie Régionale et Urbaine, 5-6 : XXXX

NADOU F, DEMAZIERE C (2018). L'aménagement à la rencontre des proximités territoriales. Application à la planification spatiale et à la coopération intercommunale en France. Revue d'Économie Régionale et Urbaine, 5-6 : XXXX

Pecqueur B, Zimmermann J-B (dir.) (2004) Economie de Proximités. Hermès, Lavoisier, Paris.

PERRAT J (2018). Les transformations de la force de travail à la lumière de l'analyse des proximités. Revue d'Économie Régionale et Urbaine, 5-6 : XXXX

Pumain D (2006), Villes et systèmes de villes dans l'économie, Revue d'Economie Financière, 86, Le financement de la ville, 29-46.

RALlet A. (2002) L'économie de proximités: Propos d'étape. Études et Recherches sur les Systèmes Agraires et le Développement, INRA Editions, 11-25.

RAllet A, TORRE A (1995) (Eds.) Economie industrielle et économie spatiale, Economica, Paris.

Rodet-KroichVili N (2018) Proximités des acteurs de la relation d'emploi et arrangements locaux d'emploi et de travail en France. Revue d'Économie Régionale et Urbaine, 5-6 : XXXX

RYCHEN F, ZIMMERMANN J-B (2008) Clusters in the Global Knowledge-based Economy: Knowledge Gatekeepers and Temporary Proximity - Introduction, Regional Studies, Special Issue, 42 6, July.

TALANDIER M, PECQUeUR B (2018) Renouveler la géographie économique. Economica, Paris. TALBOT D (2013) Clustérisation et délocalisation : les proximités construites par Thales Avionics. Revue Française de Gestion, 5(234) :15-26.

TALBOT D (2008) Les institutions créatrices de proximités. Revue d'Economie Régionale et Urbaine 3: 289-310.

TAlbot D (2018) Proximités et contrôles. Revue d'Économie Régionale et Urbaine, 5-6: XXXX

TAYLOR S, SPICER A (2007). Time for space: A narrative review of research on organizational spaces. International Journal of Management Reviews, 9(4): 325-346.

TORRE A (2018) Développement territorial et relations de proximité. Revue d'Économie Régionale et Urbaine, 5-6: XXXX 
TORRE A (2011). The role of proximity during long-distance collaborative projects.

Temporary geographical proximity helps, International Journal of Foresight and Innovation Policy, 7, 1/2/3, 213 - 230.

TORRE A (2010), Jalons pour une analyse dynamique des Proximités, Revue d'Economie Régionale et Urbaine, 3, 409-437.

TORRE A. (2009), Retour sur la notion de proximité géographique, Géographie, Economie, Société, 11, 1, 63-74.

TORRE A (2008), On the role played by temporary geographical proximity in knowledge transfer, Regional Studies, 42, 6, 869-889.

Torre A., Beuret J-E (2012). Proximités territoriales. Construire la gouvernance des territoires, entre conventions, conflits et concertations, Economica - Anthropos, Paris, 105p.

Torre A, Rallet A (2005) Proximity and Localization. Regional Studies 39(1): 47-60.

Torre A, Zuindeau B (eds) (2009). Economie de la Proximité, Nature, Sciences, Sociétés, Dossier, 17, 4 (Octobre-Décembre).

ViCENTE J, BALlAND P-A, CRESPO J (2018) Les fondements micro du changement structurel régional. Que nous enseignent 25 ans de proximités? Revue d'Économie Régionale et Urbaine, 5-6: XXXX

ZIMMERMANN J-B (1998) Firmes et territoires : du nomadisme à l'ancrage territorial. In : Bellet M, Kirat T, LARGERON C (dir.) Approches multiformes de la proximité, Hermès, Paris. 